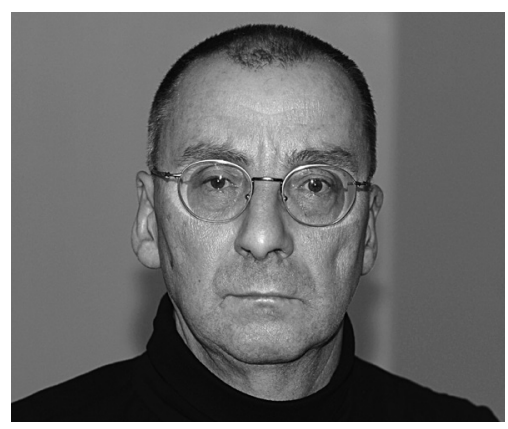

\title{
Attraktive Branche
}

Das Stolperwort der "Socioclerose" macht seit letztem Jahr die Runde in den Führungsetagen der deutschen Sozialwirtschaft. Erfunden wurde der Begriff im Institut für Arbeit und Technik der Westfälischen Universität Gelsenkirchen, Bocholt und Recklinghausen. Das Institut hatte in einem von der Europäischen Union initiierten Forschungsverbund die Zukunft des sozialen Dialogs in der Sozialwirtschaft untersucht. Im Mittelpunkt der Studie standen deren Tarif- und Arbeitsbedingungen. Das zentrale Ergebnis für Deutschland wurde in dem Befund "Socioclerose« zusammengefasst: Die Zukunftsbranche Sozialwirtschaft droht sich danach, durch zersplitterte Repräsentations- und Verhandlungsstrukturen selbst auszubremsen. Die »außergewöhnlich vielfältige Struktur des Systems der Arbeitgeber-Arbeitnehmer-Beziehungen in der deutschen Sozialwirtschaft «, so die Autoren der Studie in einer Zusammenfassung ihrer Ergebnisse in diesem Heft, stelle eine Gefährdung der Zukunftsfähigkeit der ganzen Branche dar. Während in vielen anderen Wirtschaftszweigen branchenweite Repräsentations- und Verhandlungsstrukturen dafür sorgen, dass es halbwegs einheitliche Lohnund Arbeitsbedingungen gibt und der Wettbewerb unter den Anbietern über Preise oder Qualität der Produkte und Dienstleistungen erfolge, gehe der Trend in der Sozialwirtschaft genau in die andere Richtung: Unternehmen, die in wirtschaftlichen Schwierigkeiten steckten, versuchen ihre Probleme durch Lohnabschlüsse und Arbeitsbedingungen unterhalb des bislang Branchenüblichen zu überwinden. Das einzelbetrieblich durchaus verständliche Verhalten erschwere es jedoch der Sozialwirtschaft als Ganzes außerordentlich, mittel- und langfristig für Arbeitnehmerinnen und Arbeitnehmer eine attraktive Branche zu werden - was angesichts der gewandelten Bedingungen am Arbeitsmarkt aber unerlässlich sei. Und zudem sorge die Vielfalt der Repräsentations- und Verhandlungssysteme dafür, dass die Interessen der Gesamtbranche gegenüber der Öffentlichkeit, der Politik und gegenüber anderen Branchen offensiv und einheitlich vertreten werden könnten. Viel Stoff zum Diskutieren, beispielsweise auf dem 8. Kongress der Sozialwirtschaft," der sich als zentraler Branchentreff am 13. und 14. Juni 2013 in Magdeburg genau mit diesen Fragen beschäftigen wird.

Gerhard Pfannendörfer, Chefredaktion pfannendoerfer@nomos.de

\footnotetext{
*www.sozkon.de
} 